\title{
Multiplicity of Solutions for a Modified Schrödinger-Kirchhoff-Type Equation in $\mathbb{R}^{N}$
}

\author{
Xiumei He $\mathrm{H}^{1,2}$ \\ ${ }^{1}$ Department of Mathematics and Statistics, Yunnan University, Kunming, Yunnan 650091, China \\ ${ }^{2}$ Department of Mathematics, Kunming University, Kunming, Yunnan 650214, China
}

Correspondence should be addressed to Xiumei He; hexiumei2004@163.com

Received 8 July 2015; Accepted 6 September 2015

Academic Editor: Douglas R. Anderson

Copyright (C) 2015 Xiumei He. This is an open access article distributed under the Creative Commons Attribution License, which permits unrestricted use, distribution, and reproduction in any medium, provided the original work is properly cited.

We study the existence of infinitely many solutions for a class of modified Schrödinger-Kirchhoff-type equations by the dual method and the nonsmooth critical point theory.

\section{Introduction and Main Result}

In this paper, we study the following modified SchrödingerKirchhoff-type equations of the form

$$
\begin{aligned}
& -\left(a+b \int_{\mathbb{R}^{N}}|\nabla u|^{2} d x\right) \Delta u-a u \Delta u^{2}+V(x) u \\
& =g(x, u), \quad x \in \mathbb{R}^{N},
\end{aligned}
$$

where $a>0, b \geq 0, N \geq 3, g \in C\left(\mathbb{R}^{N} \times \mathbb{R}, \mathbb{R}\right)$, and $V \in$ $C\left(\mathbb{R}^{N}, \mathbb{R}\right)$.

When $a u \Delta u^{2}=0$, (1) is reduced to the following Kirchhoff-type problem:

$$
\begin{aligned}
&-\left(a+b \int_{\mathbb{R}^{N}}|\nabla u|^{2} d x\right) \Delta u+V(x) u=g(x, u) \\
& x \in \mathbb{R}^{N} .
\end{aligned}
$$

If $V(x)=0$, problem (2) is related to the stationary analogue of the Kirchhoff equation

$$
u_{t t}-\left(a+b \int_{\mathbb{R}^{N}}|\nabla u|^{2} d x\right) \Delta u=g(x, u)
$$

proposed by Kirchhoff in [1] as an existence of the classical D'Alembert's wave equation for free vibrations of elastic string. Kirchhoff's model takes into account the changes for free vibrations of elastic strings. Recently, there have been many papers concerned with the Kirchhoff-type problems by variational methods; see [2-8] and the references therein. Many studies of them are concentrated on a bounded smooth domain $\Omega$ of $\mathbb{R}^{N}(N=1,2,3)$; it is well known that the embedding $H^{1}\left(\mathbb{R}^{N}\right) \hookrightarrow L^{t}\left(\mathbb{R}^{N}\right)\left(2 \leq t<2^{*}\right)$ is not compact. Hence, if we look for solution by variational methods, it is very difficult to prove (PS) condition. Moreover, in order to check the $(P S)$ condition or some of its variants, one has to impose certain conditions.

When $b=0$ and $a=1$, (1) is reduced to the following modified nonlinear Schrödinger equation:

$$
-\Delta u-u \Delta u^{2}+V(x) u=g(x, u), \quad x \in \mathbb{R}^{N} .
$$

Solutions of (4) are standing waves of the following quasilinear Schrödinger equations:

$$
\begin{aligned}
i \psi_{t}+ & \Delta \psi-V(x) \psi+k \Delta\left(\alpha\left(|\psi|^{2}\right)\right) \alpha^{\prime}\left(|\psi|^{2}\right) \psi \\
& +g(x, \psi)=0, \quad x \in \mathbb{R}^{\mathbb{N}},
\end{aligned}
$$

where $k$ is a real constant and $\alpha$ and $g$ are real functions. Equations (5) are derived as model of several physical phenomena, such as $[9,10]$. Many achievements had been obtained on the existence of ground states, infinitely many solutions, and soliton solutions for (4), by a dual approach, Nehari method, and the minimax methods in critical point theory, applying the perturbation approach and the Lusternik-Schnirelmann category theory; see [11-18]. 
Recently, K. Wu and X. Wu [19] obtained infinitely many small energy solutions of (1) by applying Clark's theorem to a perturbation functional. In (1), set $g(x, u)=\alpha k(x)|u|^{p-2} u+$ $\beta u^{2\left(2^{*}\right)-1}$, in which $\alpha$ and $\beta$ are real parameters, $2^{*}=2 N /(N-$ 2) $(N \geq 3)$; Liang and Shi [20] obtained soliton solutions of (1) by using the concentration-compactness principle and minimax methods.

In this paper, we transform (1) to another equation with a continuous energy functional in some Banach space. We obtain the existence of multiple solution for problem (1) via using nonsmooth critical point theory and using some new techniques. Throughout this paper, the main ideas used here come from Colin and Jeanjean [12] and Liu et al. [14].

We need the following several notations. Let

$$
H^{1}\left(\mathbb{R}^{N}\right):=\left\{u \in L^{2}\left(\mathbb{R}^{N}\right): \nabla u \in L^{2}\left(\mathbb{R}^{N}\right)\right\}
$$

with the inner product

$$
\langle u, v\rangle_{H^{1}}=\int_{\mathbb{R}^{N}}[\nabla u \cdot \nabla v+u v] d x
$$

and the norm

$$
\|u\|_{H^{1}}^{2}=\langle u, u\rangle_{H^{1}}=\int_{\mathbb{R}^{N}}\left(|\nabla u|^{2}+u^{2}\right) d x
$$

Let

$$
H_{V}^{1}\left(\mathbb{R}^{N}\right):=\left\{u \in H^{1}\left(\mathbb{R}^{N}\right): \int_{\mathbb{R}^{N}} V(x) u^{2} d x<\infty\right\}
$$

and the norm

$$
\|u\|_{H_{V}^{1}}^{2}=\int_{\mathbb{R}^{N}}\left(|\nabla u|^{2}+V(x) u^{2}\right) d x .
$$

Let the following assumption $(V)$ hold:

$(V) V \in C\left(\mathbb{R}^{N}, \mathbb{R}\right), V_{0}:=\inf _{x \in \mathbb{R}^{N}} V(x)>0$, and $\lim _{|x| \rightarrow \infty} V(x)=\infty$.

Moreover, we need the following assumptions:

$\left(g_{1}\right)$ Let $g \in C\left(\mathbb{R}^{N} \times \mathbb{R}, \mathbb{R}\right) ; g(x, t)$ is odd in $t$.

$\left(g_{2}\right)$ There exist constants $C>0$ and $4<p<22^{*}$ such that

$$
|g(x, t)| \leq C\left(1+|t|^{p-1}\right)
$$

for all $(x, t) \in \mathbb{R}^{N} \times \mathbb{R}$, where $2^{*}=2 N /(N-2)$ is the Sobolev critical exponent.

$\left(g_{3}\right) g(x, t)=o(|t|)$ uniformly in $x$ as $|t| \rightarrow 0$.

$\left(g_{4}\right)$ There exists $\mu>4$ such that $\mu G(x, t) \leq$ $g(x, t) t, \forall(x, t) \in \mathbb{R}^{N} \times \mathbb{R}$, and inf ${ }_{x \in \mathbb{R}^{\mathbb{N}},|t|=1} G(x, t)>0$, where $G(x, t):=\int_{0}^{t} g(x, s) d s$.

The main result of this paper is as follows.
Theorem 1. Assume that $(V)$ and $\left(g_{1}\right)-\left(g_{4}\right)$ are satisfied. Then problem (1) has a sequence $\left\{u_{n}\right\}$ of solutions such that $J\left(u_{n}\right) \rightarrow$ $\infty$ as $n \rightarrow \infty$.

Throughout the paper, $\rightarrow$ and $\rightarrow$ denote the strong and weak convergence, respectively. $C, c, C_{i}$, and $c_{i}$ express distinct constants. For $1 \leq s<\infty$, the usual Lebesgue space is endowed with the norm

$$
\|u\|_{s}:=\left(\int_{\mathbb{R}^{N}}|u|^{s} d x\right)^{1 / s} .
$$

The paper is organized as follows. In Section 2, we reformulate our problem into a new one which has an associated functional well defined in a suitable space and present some preliminary results. In Section 3, we introduce some notions and results of nonsmooth critical point theory and we show that the functional $I$ satisfies $(P S)_{C}$ condition. In Section 4, we complete the proof of Theorem 1 .

\section{The Dual Variational Framework and Preliminary Results}

The energy functional corresponding to problem (1) is defined as follows:

$$
\begin{aligned}
J(u)= & \frac{a}{2} \int_{\mathbb{R}^{N}}|\nabla u|^{2} d x+\frac{b}{4}\left(\int_{\mathbb{R}^{N}}|\nabla u|^{2} d x\right)^{2} \\
& +a \int_{\mathbb{R}^{N}} u^{2}|\nabla u|^{2} d x+\frac{1}{2} \int_{\mathbb{R}^{N}} V(x) u^{2} d x \\
& -\int_{\mathbb{R}^{N}} G(x, u) d x .
\end{aligned}
$$

It should be pointed out that the main difficulty in treating this class of quasilinear equations in $\mathbb{R}^{N}$ is the lack of compactness and the second-order nonhomogeneous term $a u \Delta u^{2}$ which prevents us from working directly in a classical function space. $J$ is not defined in $H_{V}^{1}\left(\mathbb{R}^{N}\right)$; thus we may not apply directly the variational method to study (1). To move these obstacles, we make the change of variable (see $[12,14]$ ); that is, we consider $v=f^{-1}(u)$, where $f$ is defined by

$$
\begin{aligned}
& f^{\prime}(t)=\frac{1}{\sqrt{1+2 f^{2}(t)}}, \quad \text { on }[0,+\infty), \\
& f(t)=-f(-t), \quad \text { on }(-\infty, 0]
\end{aligned}
$$

In order to prove our main result, we need some further properties of the function $f$.

Lemma 2. The function $f$ has the following properties:

(1) $f$ is uniquely defined, $C^{\infty}$, and invertible;

(2) $\left|f^{\prime}(t)\right| \leq 1$ for all $t \in \mathbb{R}$;

(3) $|f(t)| \leq|t|$ for all $t \in \mathbb{R}$;

(4) $\lim _{t \rightarrow 0}(f(t) / t)=1 ; \lim _{t \rightarrow+\infty}(f(t) / \sqrt{t})=2^{1 / 4}$;

(5) $|f(t)| \leq 2^{1 / 4}|t|^{1 / 2}$ for all $t \in \mathbb{R}$; 
(6) $(1 / 2) f(t) \leq t f^{\prime}(t) \leq f(t)$, for all $t \geq 0 ; f(t)<t f^{\prime}(t)<$ $(1 / 2) f(t)$ for all $t<0$;

(7) there exists a positive constant $C$ such that

$$
|f(t)| \geq \begin{cases}C|t|, & \text { if }|t| \leq 1 \\ C|t|^{1 / 2}, & \text { if }|t| \geq 1\end{cases}
$$

(8) $\left|f(t) f^{\prime}(t)\right| \leq 1 / \sqrt{2}<1$ for all $t \in \mathbb{R}$;

(9) there exist positive constants $C_{1}, C_{2}$ such that

$$
|t| \leq C_{1}|f(t)|+C_{2}|f(t)|^{2} \quad \forall t \in \mathbb{R} ;
$$

(10) $f^{2}(t)$ is convex and $f^{2}(\lambda t) \leq \lambda^{2} f^{2}(t)$ for all $t \in \mathbb{R}$ and $\lambda \geq 1$

(11) $\forall \xi>0, \exists C(\xi)>0$, s.t. $f^{2}(\xi t) \leq C(\xi) f^{2}(t)$;

(12) $f(t) / t$ is decreasing for $t>0$.

After this change of variables, the functional

$$
\begin{aligned}
I(v)= & J(f(v)) \\
= & \frac{a}{2} \int_{\mathbb{R}^{N}}|\nabla f(v)|^{2} d x+a \int_{\mathbb{R}^{N}} f^{2}(v)|\nabla f(v)|^{2} d x \\
& +\frac{b}{4}\left(\int_{\mathbb{R}^{N}}|\nabla f(v)|^{2} d x\right)^{2} \\
& +\frac{1}{2} \int_{\mathbb{R}^{N}} V(x) f^{2}(v) d x-\int_{\mathbb{R}^{N}} G(x, f(v)) d x \\
= & \frac{a}{2} \int_{\mathbb{R}^{N}}|\nabla v|^{2} d x+\frac{b}{4}\left(\int_{\mathbb{R}^{N}}|\nabla f(v)|^{2} d x\right)^{2} \\
& +\frac{1}{2} \int_{\mathbb{R}^{N}} V(x) f^{2}(v) d x-\int_{\mathbb{R}^{N}} G(x, f(v)) d x
\end{aligned}
$$

is well defined on

$$
E=\left\{v \in H^{1}\left(\mathbb{R}^{N}\right): \int_{\mathbb{R}^{N}} V(x) f^{2}(v) d x<\infty\right\}
$$

which is a Banach space endowed with the norm

$$
\begin{aligned}
\|v\| & =\|\nabla v\|_{2}+\inf _{\lambda>0} \frac{1}{\lambda}\left\{1+\int_{\mathbb{R}^{N}} V(x) f^{2}(\lambda v) d x\right\} \\
& :=\|\nabla v\|_{2}+|\|v\|| .
\end{aligned}
$$

A standard argument which is similar to that in [12] shows that if $v \in E$ is a critical point of the functional $I$, then $u=$ $f(v) \in E$ and $u$ is a weak solution of (1).

We have the following result with respect to the space $E$. Its proof can be found in $[13,14]$.
Proposition 3. (1) There exists a positive $C$ such that, for all $v \in E$,

$$
\frac{\int_{\mathbb{R}^{N}} V(x) f^{2}(v) d x}{1+\left(\int_{\mathbb{R}^{N}} V(x) f^{2}(v) d x\right)^{1 / 2}} \leq C\|v\| .
$$

(2) If $v_{n} \rightarrow v$ in $E$, then

$$
\begin{gathered}
\int_{\mathbb{R}^{N}} V(x)\left|f^{2}\left(v_{n}\right)-f^{2}(v)\right| d x \longrightarrow 0, \\
\int_{\mathbb{R}^{N}} V(x)\left|f\left(v_{n}\right)-f(v)\right|^{2} d x \longrightarrow 0 .
\end{gathered}
$$

(3) If $v_{n}(x) \rightarrow v(x)$ a.e. $x \in \mathbb{R}^{N}$ and

$$
\int_{\mathbb{R}^{N}} V(x) f^{2}\left(v_{n}\right) d x \rightarrow \int_{\mathbb{R}^{N}} V(x) f^{2}(v) d x,
$$

then

$$
\begin{aligned}
& \|\| v_{n}-v \| \mid \\
& \quad \longrightarrow 0 \text {, i.e. } \inf _{\lambda>0} \frac{1}{\lambda}\left[1+\int_{\mathbb{R}^{N}} V(x) f^{2}\left(\lambda\left(v_{n}-v\right)\right) d x\right] \\
& \longrightarrow 0 .
\end{aligned}
$$

(4) If $v_{n} \rightarrow v$ in $E$, then $f\left(v_{n}\right) \rightarrow f(v)$ in $L^{r}\left(\mathbb{R}^{N}\right)$, for $2 \leq r \leq 22^{*}$.

(5) Under assumption $(V)$, the embedding $E \hookrightarrow L^{s}\left(\mathbb{R}^{N}\right)$ is compact for $2 \leq s<2^{*}$, and the embedding $E \hookrightarrow L^{s}\left(\mathbb{R}^{N}\right)$ is continuous for $2 \leq s \leq 2^{*}$.

(6) The embedding $E \hookrightarrow H^{1}\left(\mathbb{R}^{N}\right)$ is continuous. Moreover, $C_{0}^{\infty}\left(\mathbb{R}^{N}\right)$ is dense in $E$.

Lemma 4. Assume that conditions $(V),\left(g_{2}\right)$, and $\left(g_{3}\right)$ hold; then, one has for I the following assertions:

(1) $I: E \rightarrow \mathbb{R}$ is continuous.

(2) For every $v \in E$ and $\varphi \in E \cap L^{\infty}\left(\mathbb{R}^{N}\right)$, the derivation of I in the direction $\varphi$ at $v$ exists and will be denoted by

$$
\begin{aligned}
& \left\langle I^{\prime}(v), \varphi\right\rangle=a \int_{\mathbb{R}^{N}} \nabla v \nabla \varphi d x \\
& +b\left(\int_{\mathbb{R}^{N}}\left|f^{\prime}(v)\right|^{2}|\nabla v|^{2} d x\right) \\
& \cdot\left[\int_{\mathbb{R}^{N}}\left|f^{\prime}(v)\right|^{2} \nabla v \nabla \varphi d x\right. \\
& \left.+\int_{\mathbb{R}^{N}} f^{\prime}(v) f^{\prime \prime}(v)|\nabla v|^{2} \varphi d x\right] \\
& +\int_{\mathbb{R}^{N}} V(x) f(v) f^{\prime}(v) \varphi d x \\
& -\int_{\mathbb{R}^{N}} g(x, f(v)) f^{\prime}(v) \varphi d x .
\end{aligned}
$$


(3) The map $\langle v, \varphi\rangle \rightarrow\left\langle I^{\prime}(v), \varphi\right\rangle$ satisfies the following:

(i) $\left\langle I^{\prime}(v), \varphi\right\rangle$ is linear in $\varphi \in E \cap L^{\infty}\left(\mathbb{R}^{N}\right)$;

(ii) $\left\langle I^{\prime}(v), \varphi\right\rangle$ is continuous in $v$; that is, if $v_{n} \rightarrow v$ in $E$, then $\left\langle I^{\prime}\left(v_{n}\right), \varphi\right\rangle \rightarrow\left\langle I^{\prime}(v), \varphi\right\rangle$ as $n \rightarrow \infty$.

Remark 5. Consider the following:

$\forall \varphi \in E \cap L^{\infty}\left(\mathbb{R}^{N}\right)$,

$$
\begin{array}{r}
\left\langle I^{\prime}(v), \varphi\right\rangle=a \int_{\mathbb{R}^{N}} \nabla v \nabla \varphi d x+\int_{\mathbb{R}^{N}} V(x) f(v) f^{\prime}(v) \varphi d x \\
-\int_{\mathbb{R}^{N}} g(x, f(v)) f^{\prime}(v) \varphi d x+b\left(\int_{\mathbb{R}^{N}} \frac{|\nabla v|^{2}}{1+2 f^{2}(v)} d x\right) \\
\cdot\left(\int_{\mathbb{R}^{N}} \frac{\nabla v \nabla \varphi \cdot\left(1+2 f^{2}(v)\right)-2|\nabla v|^{2} f(v) f^{\prime}(v) \varphi}{\left[1+2 f^{2}(v)\right]^{2}} d x\right) .
\end{array}
$$

Proof. Let $\left\{v_{n}\right\} \subset E$ be a sequence such that $v_{n} \rightarrow v$ in $E$. By Proposition 3, we have

$$
\lim _{n \rightarrow \infty} \int_{\mathbb{R}^{N}} V(x) f^{2}\left(v_{n}\right) d x=\int_{\mathbb{R}^{N}} V(x) f^{2}(v) d x .
$$

Moreover, $v_{n} \rightarrow v$ in $L^{s}\left(\mathbb{R}^{N}\right)$ for $2 \leq s \leq 2^{*}, \nabla v_{n} \rightarrow \nabla v$ in $L^{2}\left(\mathbb{R}^{N}\right)$, and, up to subsequence, $v_{n}(x) \rightarrow v(x), \nabla v_{n}(x) \rightarrow$ $\nabla v(x)$ a.e. $x \in \mathbb{R}^{N}$.

$\forall \varepsilon>0$, by $\left(g_{2}\right)$ and $\left(g_{3}\right)$, there exists $C_{\varepsilon}>0$ such that

$$
\begin{aligned}
& |g(x, t)| \leq \varepsilon|t|+C_{\varepsilon}|t|^{p-1}, \\
& |G(x, t)| \leq \varepsilon t^{2}+C_{\varepsilon}|t|^{p},
\end{aligned}
$$

$$
\forall(x, t) \in \mathbb{R}^{N} \times \mathbb{R} .
$$

Thus, by Lemma 2 and (27),

$$
\begin{aligned}
& \left|G\left(x, f\left(v_{n}\right)\right)-G(x, f(v))\right| \\
& \quad \leq \varepsilon\left(\left|v_{n}\right|^{2}+|v|^{2}\right)+C\left(\left|v_{n}\right|^{p / 2}+|v|^{p / 2}\right) .
\end{aligned}
$$

By Lemma A.1 of [2] and Lebesgue's theorem, one has

$$
\lim _{n \rightarrow \infty} \int_{\mathbb{R}^{N}} G\left(x, f\left(v_{n}\right)\right) d x=\int_{\mathbb{R}^{N}} G(x, f(v)) .
$$

By Lemma 2 and Lebesgue's theorem,

$$
\begin{aligned}
& \left|\left(\int_{\mathbb{R}^{N}}\left|\nabla f\left(v_{n}\right)\right|^{2} d x\right)^{2}-\left(\int_{\mathbb{R}^{N}}|\nabla f(v)|^{2} d x\right)^{2}\right| \\
& \quad \leq\left. C\left|\int_{\mathbb{R}^{N}}\right| \nabla f\left(v_{n}\right)\right|^{2} d x-\int_{\mathbb{R}^{N}}|\nabla f(v)|^{2} d x \mid \\
& \quad=\left.C\left|\int_{\mathbb{R}^{N}}\right| f^{\prime}\left(v_{n}\right)\right|^{2}\left(\left|\nabla v_{n}\right|^{2}-|\nabla v|^{2}\right) d x \\
& \quad+\int_{\mathbb{R}^{N}}\left(\left|f^{\prime}\left(v_{n}\right)\right|^{2}-\left|f^{\prime}(v)\right|^{2}\right)|\nabla v|^{2} d x \mid \leq o_{n}(1) ;
\end{aligned}
$$

thus

$$
\lim _{n \rightarrow \infty}\left(\int_{\mathbb{R}^{N}}\left|\nabla f\left(v_{n}\right)\right|^{2} d x\right)^{2}=\left(\int_{\mathbb{R}^{N}}|\nabla f(v)|^{2} d x\right)^{2} .
$$

Consequently, $I\left(v_{n}\right) \rightarrow I(v)$ and $I$ is continuous in $E$. Similarly, it can be proved that $\left\langle I^{\prime}(v), \varphi\right\rangle$ is continuous in $v$ for each $\varphi \in E \cap L^{\infty}\left(\mathbb{R}^{N}\right)$. The proof of (24) is standard by using conditions $(V),\left(g_{2}\right)$, and $\left(g_{3}\right)$. It is obvious that $\left\langle I^{\prime}(v), \varphi\right\rangle$ is linear in $\varphi \in E \cap L^{\infty}\left(\mathbb{R}^{N}\right)$. The proof is completed.

\section{Nonsmooth Critical Framework}

Let us begin by recalling some notions and results of nonsmooth critical point theory (see $[21,22]$ ).

In the following, $X$ will denote a metric space endowed with the metric $d$.

Definition 6. Let $f: X \rightarrow \mathbb{R}$ be a continuous function and let $u \in X$. One denotes by $|d f|(u)$ the supremum of the $\sigma$ 's in $[0,+\infty)$ such that there exist $\delta>0$ and a continuous map

$$
H: B(u, \delta) \times[0, \delta] \longrightarrow X
$$

satisfying

$$
\begin{aligned}
d(H(v, t), v) & \leq t, \\
f(H(v, t)) & \leq f(v)-\sigma t
\end{aligned}
$$

for all $(v, t) \in B(u, \delta) \times[0, \delta]$, where $B(u, \delta)$ is the open ball of center $u \in X$ and of radius $\delta$. The extended real number $|d f|(u)$ is called the weak slope of $f$ at $u$.

If $X$ is a Finsler manifold of class $C^{1}$ and $f \in C^{1}$, it turns out that $|d f|(u)=\left\|f^{\prime}(u)\right\|$.

The function $|d f|: X \rightarrow[0,+\infty]$ is lower semicontinuous.

Definition 7. Let $f: X \rightarrow \mathbb{R}$ be a continuous function. A point $u \in X$ is called a critical point of $f$ if $|d f|(u)=0$. A real number $c$ is called a critical value of $f$ if there exists a $u \in X$ such that $f(u)=c$ and $|d f|(u)=0$.

Definition 8. Let $f: X \rightarrow \mathbb{R}$ be a continuous function and let $c \in \mathbb{R}$. One says that $f$ satisfies the $(P S)_{c}$ condition if each sequence $\left\{u_{n}\right\} \subset X$ with $f\left(u_{n}\right) \rightarrow c$ and $|d f|\left(u_{n}\right) \rightarrow 0$ has a convergent subsequence.

We can state a generalized version of the symmetric Mountain Pass Theorem for the case of continuous functionals. 
Theorem 9 (see [21]). Let $E$ be an infinite-dimensional Banach space and let $f: E \rightarrow \mathbb{R}$ be continuous, even, and satisfying $(P S)_{c}$ condition for every $c \in \mathbb{R}$. Assume the following:

(i) There exist $\rho>0, \beta>f(0)$, and a subspace $V \subset E$ of finite codimension such that

$$
\forall u \in V:\|u\|=\rho \Longrightarrow f(u) \geq \beta \text {. }
$$

(ii) For every finite-dimensional subspace $W \subset E$, there exists $R>0$ such that

$$
\forall u \in W:\|u\| \geq R \Longrightarrow f(u) \leq f(0) .
$$

Then there exists a sequence $\left\{c_{n}\right\}$ of critical values of $f$ with $c_{n} \rightarrow \infty$ as $n \rightarrow \infty$.

Now consider the functional $I$ given in the previous section.

Lemma 10. Consider $|d I|(v) \geq \sup \left\{\left\langle I^{\prime}(v), \varphi\right\rangle: \varphi \in E \cap\right.$ $\left.L^{\infty}\left(\mathbb{R}^{N}\right),\|\varphi\|=1\right\}$.

Proof. We use a similar argument in the proof of Theorem 1.5 in [21]. Let $v \in E$ and

$$
\begin{aligned}
& \Psi(v) \\
& \quad=\sup \left\{\left\langle I^{\prime}(v), \varphi\right\rangle: \varphi \in E \cap L^{\infty}\left(\mathbb{R}^{N}\right),\|\varphi\|=1\right\} .
\end{aligned}
$$

If $\Psi(v)=0$, the conclusion is obvious. Otherwise, take $\sigma>0$ such that $\Psi(v)>\sigma$. Then there exists a point $\varphi \in E \cap L^{\infty}\left(\mathbb{R}^{N}\right)$ with $\|\varphi\|=1$ and $\left\langle I^{\prime}(v), \varphi\right\rangle>\sigma$. By Lemma 4 , there exist $\delta_{0}>0$ such that $\left\langle I^{\prime}(u), \varphi\right\rangle>\sigma$ for every $u \in B\left(v, \delta_{0}\right)$. Let $\delta=\delta_{0} / 2$ and $H(u, t)=u-t \varphi$. It is obvious that $H: B(u, \delta) \times$ $[0, \delta] \rightarrow E$ is continuous,

$$
\begin{aligned}
\|H(u, t)-u\| \leq & t \\
I(H(u, t))= & I(H(u, 0)) \\
& -t \int_{0}^{1}\left\langle I^{\prime}(H(u, s t)), \varphi\right\rangle d s \\
& \leq I(u)-\sigma t
\end{aligned}
$$

for $(u, t) \in B(v, \delta) \times[0, \delta]$. Hence, by Definition $6,|d I|(v) \geq$ $\sigma$ and $|d I|(v) \geq \Psi(v)$ by the arbitrariness of $\sigma$. The proof is completed.

Lemma 11. Under assumptions $\left(g_{2}\right),\left(g_{3}\right)$, and $(V)$, if $v \in E$ satisfies $\left\langle I^{\prime}(v), \varphi\right\rangle=0, \forall \varphi \in E \cap L^{\infty}\left(\mathbb{R}^{N}\right)$, then $v \in L^{\infty}\left(\mathbb{R}^{N}\right)$. Proof. By (25), we have

$$
\begin{aligned}
& a \int_{\mathbb{R}^{N}} \nabla v \nabla \varphi d x+b\left(\int_{\mathbb{R}^{N}} \frac{|\nabla v|^{2}}{1+2 f^{2}(v)} d x\right) \\
& \quad \cdot\left(\int_{\mathbb{R}^{N}} \frac{\nabla v \nabla \varphi \cdot\left(1+2 f^{2}(v)\right)-2|\nabla v|^{2} f(v) f^{\prime}(v) \varphi}{\left[1+2 f^{2}(v)\right]^{2}} d x\right) \\
& \quad=\int_{\mathbb{R}^{N}} g(x, f(v)) f^{\prime}(v) \varphi d x \\
& -\int_{\mathbb{R}^{N}} V(x) f(v) f^{\prime}(v) \varphi d x
\end{aligned}
$$

for all $\varphi \in E \cap L^{\infty}\left(\mathbb{R}^{N}\right)$.

For any $T>0$, let $v^{T}=v$ if $|v| \leq T$ and $v^{T}=\operatorname{sign}(v) T$ if $|v| \geq T$. For any $r>0$, take $\varphi=v\left|v^{T}\right|^{2 r}$ in the above equality; then it can be deduced from (38) that

$$
\begin{aligned}
& a \int_{\mathbb{R}^{N}} \nabla v \cdot \nabla\left(v\left|v^{T}\right|^{2 r}\right) d x \\
& \quad+b\left(\int_{\mathbb{R}^{N}} \frac{|\nabla v|^{2}}{1+2 f^{2}(v)} d x\right)\left(\int_{\mathbb{R}^{N}} \frac{\nabla v \cdot \nabla\left(v\left|v^{T}\right|^{2 r}\right)\left(1+2 f^{2}(v)\right)-2|\nabla v|^{2} f(v) f^{\prime}(v) \cdot v\left|v^{T}\right|^{2 r}}{\left[1+2 f^{2}(v)\right]^{2}} d x\right) \\
& \quad=\int_{\mathbb{R}^{N}} g(x, f(v)) f^{\prime}(v) \cdot v\left|v^{T}\right|^{2 r} d x-\int_{\mathbb{R}^{N}} V(x) f(v) f^{\prime}(v) \cdot v\left|v^{T}\right|^{2 r} d x .
\end{aligned}
$$

By Lemma 2 and the Sobolev embedding theorem,

$$
\begin{aligned}
& a \int_{\mathbb{R}^{N}} \nabla v \cdot \nabla\left(v\left|v^{T}\right|^{2 r}\right) d x \\
& \quad+b\left(\int_{\mathbb{R}^{N}} \frac{|\nabla v|^{2}}{1+2 f^{2}(v)} d x\right)\left(\int_{\mathbb{R}^{N}} \frac{\nabla v \cdot \nabla\left(v\left|v^{T}\right|^{2 r}\right)\left(1+2 f^{2}(v)\right)-2|\nabla v|^{2} f(v) f^{\prime}(v) \cdot v\left|v^{T}\right|^{2 r}}{\left[1+2 f^{2}(v)\right]^{2}} d x\right) \\
& \quad \geq a \int_{\mathbb{R}^{N}} \nabla v \cdot \nabla\left(v\left|v^{T}\right|^{2 r}\right) d x=a \int_{\mathbb{R}^{N}}\left[|\nabla v|^{2}\left|v^{T}\right|^{2 r}+2 r|v|^{2 r}\left|\nabla v^{T}\right|^{2}\right] d x
\end{aligned}
$$




$$
\begin{aligned}
& \geq \frac{a}{r+2} \int_{\mathbb{R}^{N}}\left[|\nabla v|^{2}\left|v^{T}\right|^{2 r}+\left(2 r+r^{2}\right)|v|^{2 r}\left|\nabla v^{T}\right|^{2}\right] d x=\frac{a}{r+2} \int_{\mathbb{R}^{N}}\left|\nabla\left(v\left|v^{T}\right|^{r}\right)\right|^{2} d x \\
& \geq \frac{C_{1}}{r+1}\left(\left.\left.\int_{\mathbb{R}^{N}}|v \cdot| v^{T}\right|^{r}\right|^{2^{*}} d x\right)^{2 / 2^{*}},
\end{aligned}
$$

where $C_{1}$ is positive constant.

For any $0<\varepsilon \leq V_{0}$, by (27), $(V)$, Lemma 2, Hölder inequality, and Sobolev inequality, for simplicity, taking $q=$ $p / 2$, we have

$$
\begin{aligned}
& \int_{\mathbb{R}^{N}} g(x, f(v)) f^{\prime}(v) \cdot v\left|v^{T}\right|^{2 r} d x \\
& -\int_{\mathbb{R}^{N}} V(x) f(v) f^{\prime}(v) \cdot v\left|v^{T}\right|^{2 r} d x \\
& \leq \int_{\mathbb{R}^{N}}\left(\varepsilon|f(v)|+C_{\varepsilon}|f(v)|^{p-1}\right)\left|f^{\prime}(v) v\right| \\
& \cdot\left|v^{T}\right|^{2 r} d x-\int_{\mathbb{R}^{N}} V_{0}\left|f(v) f^{\prime}(v) v\right| \cdot\left|v^{T}\right|^{2 r} d x \\
& \leq C \int_{\mathbb{R}^{N}}|f(v)|^{p} \cdot\left|v^{T}\right|^{2 r} d x \leq C \int_{\mathbb{R}^{N}}|v|^{p / 2} \\
& \cdot\left|v^{T}\right|^{2 r} d x \\
& \quad \leq C\left[\int_{\mathbb{R}^{N}}\left(|v|^{2}\left|v^{T}\right|^{2 r}\right)^{2^{*} /\left(2^{*}+2-q\right)} d x\right]^{\left(2^{*}+2-q\right) / 2^{*}} \\
& \quad\left[\int_{\mathbb{R}^{N}}|v|^{2^{*}} d x\right]^{(q-2) / 2^{*}} \\
& \leq C_{2}\left[\int_{\mathbb{R}^{N}}\left(|v|^{2}\left|v^{T}\right|^{2 r}\right)^{2^{*} /\left(2^{*}+2-q\right)} d x\right]^{\left(2^{*}+2-q\right) / 2^{*}}
\end{aligned}
$$

where $C$ and $C_{2}$ are positive constants.

Set $d=2^{*} /\left(2^{*}+2-q\right)$. Because $2<q=p / 2<2^{*}$, $1<d<2^{*} / 2$. Moreover, by (39), (40), and (41), we have

$$
\begin{aligned}
& \left(\int_{\mathbb{R}^{N}}|v|^{2^{*}}\left|v^{T}\right|^{2^{*} r} d x\right)^{1 / 2^{*}(r+1)} \\
& \quad \leq[C(r+1)]^{1 / 2(r+1)}\left(\int_{\mathbb{R}^{N}}|v|^{2 d}\left|v^{T}\right|^{2 d r} d x\right)^{1 / 2 d(r+1)}
\end{aligned}
$$

Taking the limit $T \rightarrow \infty$ in (42),

$$
\begin{aligned}
& \left(\int_{\mathbb{R}^{N}}|v|^{2^{*}(r+1)} d x\right)^{1 / 2^{*}(r+1)} \\
& \quad \leq[C(r+1)]^{1 / 2(r+1)}\left(\int_{\mathbb{R}^{N}}|v|^{2 d(r+1)} d x\right)^{1 / 2 d(r+1)} .
\end{aligned}
$$

Set $r_{0}=0$ and $2 d\left(r_{k+1}+1\right)=2^{*}\left(r_{k}+1\right), \forall k \in \mathbb{N}$. Then $r_{k} \rightarrow \infty$ as $k \rightarrow \infty$ and

$$
\begin{aligned}
& \left(\int_{\mathbb{R}^{N}}|v|^{2 d\left(r_{k}+1\right)} d x\right)^{1 / 2 d\left(r_{k}+1\right)} \\
& \quad \leq \prod_{i=1}^{k}\left[C\left(r_{i}+1\right)\right]^{1 / 2\left(r_{i}+1\right)}\left(\int_{\mathbb{R}^{N}}|v|^{2^{*}} d x\right)^{1 / 2^{*}} .
\end{aligned}
$$

Because

$$
\begin{aligned}
& \prod_{i=1}^{k}\left[C\left(r_{i}+1\right)\right]^{1 / 2\left(r_{i}+1\right)} \\
& \quad=\exp \left\{\sum_{i=1}^{k} \frac{\ln C\left(\left(2^{*} / 2\right) d^{-1}\right)^{i}}{2\left(\left(2^{*} / 2\right) d^{-1}\right)^{i}}\right\} \\
& \quad=\exp \left\{\sum_{i=1}^{k}\left[\frac{\ln C}{2\left(\left(2^{*} / 2\right) d^{-1}\right)^{i}}+\frac{i \ln \left(\left(2^{*} / 2\right) d^{-1}\right)}{2\left(\left(2^{*} / 2\right) d^{-1}\right)^{i}}\right]\right\}
\end{aligned}
$$

is convergent as $k \rightarrow \infty$, let $C_{k}=\prod_{i=1}^{k}\left[C\left(r_{i}+1\right)\right]^{1 / 2\left(r_{i}+1\right)}$; then $C_{k} \rightarrow C_{\infty}>0$ as $k \rightarrow \infty$. Hence

$$
\|v\|_{L^{2 d\left(r_{k}+1\right)}\left(\mathbb{R}^{\mathbb{N}}\right)} \leq C_{k}\|v\|_{L^{2^{*}}\left(\mathbb{R}^{\mathbb{N}}\right)} .
$$

Let $k \rightarrow \infty$; then, we have

$$
\|v\|_{L^{\infty}\left(\mathbb{R}^{\mathbb{N}}\right)} \leq C_{\infty}\|v\|_{L^{2^{*}}\left(\mathbb{R}^{\mathbb{N}}\right)} \leq C
$$

where the positive constant $C$ is independent of $k$. The proof is completed.

Corollary 12. If $v \in E$ is a critical point of $I$, then $v \in L^{\infty}\left(\mathbb{R}^{N}\right)$.

Proof. The proof is completed by Lemmas 10 and 11 .

Lemma 13. $\forall c \in \mathbb{R}$, under assumptions $\left(g_{2}\right),\left(g_{3}\right)$, and $\left(g_{4}\right)$, the functional I satisfies $(P S)_{c}$ condition.

Proof. Let $\left\{v_{n}\right\} \subset E$ be a sequence with $I\left(v_{n}\right) \rightarrow c$ and $|d I|\left(v_{n}\right) \rightarrow 0$. Then, it follows from Lemma 10 that

$$
\begin{aligned}
\delta_{n} & =\sup \left\{\left\langle I^{\prime}\left(v_{n}\right), \varphi\right\rangle: \varphi \in E \cap L^{\infty}\left(\mathbb{R}^{N}\right),\|\varphi\|=1\right\} \\
& \longrightarrow 0
\end{aligned}
$$

Moreover,

$$
\left|\left\langle I^{\prime}\left(v_{n}\right), \varphi\right\rangle\right| \leq \delta_{n}\|\varphi\|, \quad \forall \varphi \in E \cap L^{\infty}\left(\mathbb{R}^{N}\right) .
$$


Note that

$$
\begin{aligned}
\left\langle I^{\prime}\left(v_{n}\right), \varphi\right\rangle= & a \int_{\mathbb{R}^{N}} \nabla v_{n} \nabla \varphi d x+\int_{\mathbb{R}^{N}} V(x) f\left(v_{n}\right) f^{\prime}\left(v_{n}\right) \varphi d x-\int_{\mathbb{R}^{N}} g\left(x, f\left(v_{n}\right)\right) f^{\prime}\left(v_{n}\right) \varphi d x \\
& +b\left(\int_{\mathbb{R}^{N}} \frac{\left|\nabla v_{n}\right|^{2}}{1+2 f^{2}\left(v_{n}\right)} d x\right)\left(\int_{\mathbb{R}^{N}} \frac{\nabla v_{n} \nabla \varphi \cdot\left(1+2 f^{2}\left(v_{n}\right)\right)-2\left|\nabla v_{n}\right|^{2} f\left(v_{n}\right) f^{\prime}\left(v_{n}\right) \varphi}{\left[1+2 f^{2}\left(v_{n}\right)\right]^{2}} d x\right) .
\end{aligned}
$$

Letting $\varphi=w_{n}=f\left(v_{n}\right) / f^{\prime}\left(v_{n}\right)=\sqrt{1+2 f^{2}\left(v_{n}\right)} f\left(v_{n}\right)$ and

$$
\left|\nabla w_{n}\right|=\left(1+\frac{2 f^{2}\left(v_{n}\right)}{1+2 f^{2}\left(v_{n}\right)}\right)\left|\nabla v_{n}\right| \leq 2\left|\nabla v_{n}\right|,
$$

we deduce that $\left\|w_{n}\right\| \leq C\left\|v_{n}\right\|$ for some positive constant $C$. From (49) and (50), we have

$$
\begin{aligned}
C \delta_{n}\left\|v_{n}\right\| \geq & a \int_{\mathbb{R}^{N}}\left(1+\frac{2 f^{2}\left(v_{n}\right)}{1+2 f^{2}\left(\mathrm{v}_{n}\right)}\right)\left|\nabla v_{n}\right|^{2} d x \\
& +b\left(\int_{\mathbb{R}^{N}} \frac{\left|\nabla v_{n}\right|^{2}}{1+2 f^{2}\left(v_{n}\right)} d x\right)^{2} \\
& +\int_{\mathbb{R}^{N}} V(x) f^{2}\left(v_{n}\right) d x \\
& -\int_{\mathbb{R}^{N}} g\left(x, f\left(v_{n}\right)\right) f\left(v_{n}\right) d x
\end{aligned}
$$

for each $n$. Since $I\left(v_{n}\right) \rightarrow c$, by $\left(g_{4}\right)$ and Lemma $2, \forall \lambda>0$, we have

$$
\begin{aligned}
c & +o_{n}(1)+C \delta_{n}\left\|v_{n}\right\| \geq I\left(v_{n}\right)-\frac{1}{\mu}\left\langle I^{\prime}\left(v_{n}\right), w_{n}\right\rangle \\
& =a \int_{\mathbb{R}^{N}}\left[\frac{1}{2}-\frac{1}{\mu}\left(1+\frac{2 f^{2}\left(v_{n}\right)}{1+2 f^{2}\left(v_{n}\right)}\right)\right]\left|\nabla v_{n}\right|^{2} d x \\
& +\left(\frac{1}{4}-\frac{1}{\mu}\right) b\left(\int_{\mathbb{R}^{N}}\left|f^{\prime}\left(v_{n}\right)\right|^{2}\left|\nabla v_{n}\right|^{2} d x\right)^{2}+\left(\frac{1}{2}\right. \\
& \left.-\frac{1}{\mu}\right) \int_{\mathbb{R}^{N}} V(x) f^{2}\left(v_{n}\right) d x \\
& +\int_{\mathbb{R}^{N}}\left[\frac{1}{\mu} g\left(x, f\left(v_{n}\right)\right) f\left(v_{n}\right)-G\left(x, f\left(v_{n}\right)\right)\right] d x \\
& \geq a\left(\frac{1}{2}-\frac{2}{\mu}\right) \int_{\mathbb{R}^{N}}\left|\nabla v_{n}\right|^{2} d x+\left(\frac{1}{2}-\frac{1}{\mu}\right) \int_{\mathbb{R}^{N}} V(x) \\
& +f^{2}\left(v_{n}\right) d x \geq C_{1}\left[\int_{\mathbb{R}^{N}}\left|\nabla v_{n}\right|^{2} d x+1\right. \\
& \left.+\int_{\mathbb{R}^{N}} V(x) f^{2}\left(v_{n}\right)\right] d x-C_{1} \geq C_{1}\left[\int_{\mathbb{R}^{N}}\left|\nabla v_{n}\right|^{2} d x\right. \\
& \left.+\inf _{\lambda>0} \frac{1}{\lambda}\left\{1+\int_{\mathbb{R}^{N}} V(x) f^{2}(\lambda v) d x\right\}\right]-C_{1},
\end{aligned}
$$

where $C_{1}>0$. Their estimates imply that $v_{n}$ is bounded in $E$.
Hence, up to a subsequence, $v_{n} \rightarrow v$ in $E$. Because $\delta_{n} \rightarrow$ 0 , from (49) and (50),

$$
\begin{aligned}
o_{n}(1) & +\int_{\mathbb{R}^{N}} g\left(x, f\left(v_{n}\right)\right) f\left(v_{n}\right) d x \\
= & a \int_{\mathbb{R}^{N}}\left(1+\frac{2 f^{2}\left(v_{n}\right)}{1+2 f^{2}\left(v_{n}\right)}\right)\left|\nabla v_{n}\right|^{2} d x \\
& +b\left(\int_{\mathbb{R}^{N}}\left|f^{\prime}\left(v_{n}\right)\right|^{2}\left|\nabla v_{n}\right|^{2} d x\right)^{2} \\
& +\int_{\mathbb{R}^{N}} V(x) f^{2}\left(v_{n}\right) d x .
\end{aligned}
$$

Because $\left\langle I^{\prime}(v), \varphi\right\rangle=0$ for each $\varphi \in E \cap L^{\infty}\left(\mathbb{R}^{N}\right)$, set $w=$ $f(v) / f^{\prime}(v)$; it follows from Lemma 11 that $\left\langle I^{\prime}(v), w\right\rangle=0$. Consequently,

$$
\begin{aligned}
\int_{\mathbb{R}^{N}} g(x, f(v)) f(v) d x \\
=a \int_{\mathbb{R}^{N}}\left(1+\frac{2 f^{2}(v)}{1+2 f^{2}(v)}\right)|\nabla v|^{2} d x \\
\quad+b\left(\int_{\mathbb{R}^{N}}\left|f^{\prime}(v)\right|^{2}|\nabla v|^{2} d x\right)^{2} \\
\quad+\int_{\mathbb{R}^{N}} V(x) f^{2}(v) d x .
\end{aligned}
$$

By Proposition 3, we get $v_{n} \rightarrow v$ in $L^{s}\left(\mathbb{R}^{N}\right)$ for $2 \leq s<2^{*}$. Using (27), Lemma 2 , and Lebesgue's theorem, we obtain

$$
\begin{aligned}
& \int_{\mathbb{R}^{N}} g\left(x, f\left(v_{n}\right)\right) f\left(v_{n}\right) d x \\
& \quad \longrightarrow \int_{\mathbb{R}^{N}} g(x, f(v)) f(v) d x .
\end{aligned}
$$


By assumption $(V)$, Lemma 2, the Fatou Lemma, and the Lebesgue dominated convergence theorem, one has

$$
\begin{aligned}
& \liminf _{n \rightarrow \infty} \int_{\mathbb{R}^{N}}\left|\nabla v_{n}\right|^{2} d x \geq \int_{\mathbb{R}^{N}}|\nabla v|^{2} d x, \\
& \liminf _{n \rightarrow \infty} \int_{\mathbb{R}^{N}} \frac{2 f^{2}\left(v_{n}\right)}{1+2 f^{2}\left(v_{n}\right)}\left|\nabla v_{n}\right|^{2} d x \\
& \geq \int_{\mathbb{R}^{N}} \frac{2 f^{2}(v)}{1+2 f^{2}(v)}|\nabla v|^{2} d x, \\
& \liminf _{n \rightarrow \infty} \int_{\mathbb{R}^{N}}\left|f^{\prime}\left(v_{n}\right)\right|^{2}\left|\nabla v_{n}\right|^{2} d x \\
& \quad \int_{\mathbb{R}^{N}}\left|f^{\prime}(v)\right|^{2}|\nabla v|^{2} d x, \\
& \liminf _{n \rightarrow \infty} \int_{\mathbb{R}^{N}} V(x) f^{2}\left(v_{n}\right) d x \geq \int_{\mathbb{R}^{N}} V(x) f^{2}(v) d x .
\end{aligned}
$$

These imply that

$$
\begin{gathered}
\int_{\mathbb{R}^{N}}\left|\nabla v_{n}\right|^{2} d x \rightarrow \int_{\mathbb{R}^{N}}|\nabla v|^{2} d x, \\
\int_{\mathbb{R}^{N}} V(x) f^{2}\left(v_{n}\right) d x \rightarrow \int_{\mathbb{R}^{N}} V(x) f^{2}(v) d x .
\end{gathered}
$$

Notice that $\nabla v_{n} \rightarrow \nabla v$ in $L^{2}\left(\mathbb{R}^{N}\right)$; then, we conclude that $\nabla v_{n} \rightarrow \nabla v$ in $L^{2}\left(\mathbb{R}^{N}\right)$. By Proposition 3 and (59), we have

$$
\inf _{\lambda>0} \frac{1}{\lambda}\left[1+\int_{\mathbb{R}^{N}} V(x) f^{2}\left(\lambda\left(v_{n}-v\right)\right) d x\right] \longrightarrow 0 .
$$

We obtain $v_{n} \rightarrow v$ in $E$; that is, the functional $I$ satisfies $(P S)_{C}$ condition.

\section{Proof of the Main Result}

The following lemma implies that $I$ possesses the symmetric Mountain Pass Geometry.

Lemma 14. Suppose that $(V),\left(g_{2}\right)$, and $\left(g_{3}\right)$ are satisfied. Then the functional I satisfies condition (i) of Theorem 9.

Proof. Let $S(\rho)=\{v \in E:\|v\|=\rho\}, 0<\rho<4$. If $|\|v\|| \geq$ $(1 / 2) \rho$, by Lemma 2 , we have

$$
\begin{aligned}
\frac{1}{2} \rho & \leq \inf _{\lambda>0} \frac{1}{\lambda}\left\{1+\int_{\mathbb{R}^{N}} V(x) f^{2}(\lambda v) d x\right\} \\
& \leq \frac{1}{4} \rho+\frac{1}{4} \rho \int_{\mathbb{R}^{N}} V(x) f^{2}\left(\frac{4}{\rho} v\right) d x \\
& \leq \frac{1}{4} \rho+\frac{4}{\rho} \int_{\mathbb{R}^{N}} V(x) f^{2}(v) d x,
\end{aligned}
$$

which implies that

$$
\int_{\mathbb{R}^{N}}\left(|\nabla v|^{2}+V(x) f^{2}(v)\right) d x \geq \frac{1}{16} \rho^{2}
$$

for $|\|v\|| \geq(1 / 2) \rho$. Note that $\|\nabla v\|_{2} \geq(1 / 2) \rho$ if $v \in S(\rho)$ with $|\|v\||<(1 / 2) \rho$. Hence

$$
\inf _{v \in S(\rho)} \int_{\mathbb{R}^{N}}\left(|\nabla v|^{2}+V(x) f^{2}(v)\right) d x \geq \frac{1}{16} \rho^{2} .
$$

By $(V),(27)$, Lemma 2, and Proposition 3, let $0<\varepsilon \leq(1 / 4) V_{0}$; then, we have

$$
\begin{aligned}
I(v) \geq & \frac{a}{2} \int_{\mathbb{R}^{N}}|\nabla v|^{2} d x+\frac{1}{2} \int_{\mathbb{R}^{N}} V(x) f^{2}(v) d x \\
& -\int_{\mathbb{R}^{N}}\left(\varepsilon f^{2}(v)+C_{\varepsilon}|v|^{p}\right) d x \\
\geq & \frac{a}{2} \int_{\mathbb{R}^{N}}|\nabla v|^{2} d x+\frac{1}{4} \int_{\mathbb{R}^{N}} V(x) f^{2}(v) d x \\
& -C \int_{\mathbb{R}^{N}}|v|^{p / 2} d x \\
\geq & \frac{1}{16} \min \left\{\frac{a}{2}, \frac{1}{4}\right\} \rho^{2}-C \rho^{p / 2}
\end{aligned}
$$

for $v \in S(\rho)$ and $\rho<1$. Hence condition (i) in Theorem 9 holds for small $\rho>0$.

Lemma 15. Assume that $(V),\left(g_{2}\right)$, and $\left(g_{4}\right)$ hold; then the functional I satisfies condition (ii) of Theorem 9.

Proof. By Lemma 2, for $m>4, \exists C>0$, such that

$$
|t|^{m / 2} \leq t^{2}+C|f(t)|^{m}, \quad \forall t \in \mathbb{R} .
$$

By (27) and $\left(g_{4}\right), \exists C_{1}>0$ and $C_{2}>0$; one has

$$
G(x, s) \geq C_{1}|s|^{\mu}-C_{2} s^{2}, \quad \forall(x, s) \in \mathbb{R}^{N} \times \mathbb{R} .
$$

By Lemma 2, we have

$$
\begin{aligned}
G(x, f(s)) & \geq C_{1}|f(s)|^{\mu}-C_{2} f^{2}(s) \\
& \geq C_{3}|s|^{\mu / 2}-C_{4} s^{2}
\end{aligned}
$$

$\forall(x, s) \in \mathbb{R}^{N} \times \mathbb{R}, C_{3}>0, C_{4}>0$. Let $W$ be any finitedimensional subspace of $E, \forall v \in W$; by (65), (67), and Lemma 2,

$$
\begin{aligned}
I(v) \leq & C\left(\int_{\mathbb{R}^{N}}|\nabla v|^{2} d x+\int_{\mathbb{R}^{N}} V(x) v^{2} d x\right) \\
& +C \int_{\mathbb{R}^{N}}|v|^{2} d x-\int_{\mathbb{R}^{N}} G(x, f(v)) d x \\
\leq & C \int_{\mathbb{R}^{N}}\left(|\nabla v|^{2}+V(x) v^{2}\right) d x+C \int_{\mathbb{R}^{N}}|v|^{2} d x \\
& -C_{3} \int_{\mathbb{R}^{N}}|v|^{\mu / 2} d x+C_{4} \int_{\mathbb{R}^{N}} v^{2} d x .
\end{aligned}
$$

Since all norms are equivalent in a finite-dimensional space $W, I(v) \rightarrow-\infty$ as $\|v\| \rightarrow \infty$. So the conclusion follows.

Proof of Theorem 1. The functional $I$ is evidently even. Therefore, Theorem 9 implies that $I$ has a critical sequence $\left\{v_{n}\right\} \subset E$ such that $I\left(v_{n}\right) \rightarrow \infty$ as $n \rightarrow \infty$. Note that $I\left(v_{n}\right)=J\left(f\left(v_{n}\right)\right)$; the proof is completed. 


\section{Conflict of Interests}

The author declares that there is no conflict of interests regarding the publication of this paper.

\section{Acknowledgments}

This work is supported by Scientific Research Foundation of Yunnan Province Education Department (2015C075Y) and the Scholarship Award for Excellent Doctoral Student Granted by Yunnan Province.

\section{References}

[1] G. Kirchhoff, Mechanik, Teubner, Leipzig, Germany, 1883.

[2] S.-J. Chen and L. Li, "Multiple solutions for the nonhomogeneous Kirchhoff equation on $\mathbb{R}^{N}$," Nonlinear Analysis. Real World Applications, vol. 14, no. 3, pp. 1477-1486, 2013.

[3] X. M. He and W. M. Zou, "Infinitely many positive solutions for Kirchhoff-type problems," Nonlinear Analysis: Theory, Methods \& Applications, vol. 70, no. 3, pp. 1407-1414, 2009.

[4] J. Jin and X. Wu, "Infinitely many radial solutions for Kirchhofftype problems in $\mathbb{R}^{N}$," Journal of Mathematical Analysis and Applications, vol. 369, no. 2, pp. 564-574, 2010.

[5] Q. Q. Li and X. Wu, "A new result on high energy solutions for Schrödinger-Kirchhoff type equations in $\mathbb{R}^{N}$," Applied Mathematics Letters, vol. 30, pp. 24-27, 2014.

[6] J. J. Nie, "Existence and multiplicity of nontrivial solutions for a class of Schrödinger-Kirchhoff-type equations," Journal of Mathematical Analysis and Applications, vol. 417, no. 1, pp. 6579, 2014.

[7] J. Sun and T.-f. Wu, "Ground state solutions for an indefinite Kirchhoff type problem with steep potential well," Journal of Differential Equations, vol. 256, no. 4, pp. 1771-1792, 2014.

[8] Y. W. Ye and C. L. Tang, "Multiple solutions for Kirchhoff-type equations in $\mathbb{R}^{N}$," Journal of Mathematical Physics, vol. 54, no. 8, Article ID 081508, 2013.

[9] S. Kurihara, "Large-amplitude quasi-solitons in superfluid films," Journal of the Physical Society of Japan, vol. 50, no. 10, pp. 3262-3267, 1981.

[10] E. W. Laedke, K. H. Spatschek, and L. Stenflo, "Evolution theorem for a class of perturbed envelope soliton solutions," Journal of Mathematical Physics, vol. 24, no. 12, pp. 2764-2769, 1983.

[11] J. F. L. Aires and M. A. S. Souto, "Existence of solutions for a quasilinear Schrödinger equation with vanishing potentials," Journal of Mathematical Analysis and Applications, vol. 416, no. 2, pp. 924-946, 2014.

[12] M. Colin and L. Jeanjean, "Solutions for a quasilinear Schrödinger equation: a dual approach," Nonlinear Analysis: Theory, Methods \& Applications, vol. 56, no. 2, pp. 213-226, 2004.

[13] J. M. do Ó and U. Severo, "Quasilinear Schrödinger equations involving concave and convex nonlinearities," Communications on Pure and Applied Analysis, vol. 8, no. 2, pp. 621-644, 2009.

[14] J.-Q. Liu, Y.-Q. Wang, and Z.-Q. Wang, "Soliton solutions for quasilinear Schrödinger equations, II," Journal of Differential Equations, vol. 187, no. 2, pp. 473-493, 2003.
[15] J.-Q. Liu, Y.-Q. Wang, and Z.-Q. Wang, "Solutions for quasilinear schrödinger equations via the nehari method," Communications in Partial Differential Equations, vol. 29, no. 5-6, pp. 879901, 2004.

[16] Y. Wu and T. An, "Infinitely many solutions for a class of semilinear elliptic equations," Journal of Mathematical Analysis and Applications, vol. 414, no. 1, pp. 285-295, 2014.

[17] Y. W. Ye and C.-L. Tang, "Infinitely many solutions for fourthorder elliptic equations," Journal of Mathematical Analysis and Applications, vol. 394, no. 2, pp. 841-854, 2012.

[18] F. Zhou and K. Wu, "Infinitely many small solutions for a modified nonlinear Schrödinger equations," Journal of Mathematical Analysis and Applications, vol. 411, no. 2, pp. 953-959, 2014.

[19] K. Wu and X. Wu, "Infinitely many small energy solutions for a modified Kirchhoff-type equation in $\mathbb{R}^{N}$," Computers \& Mathematics with Applications, vol. 70, no. 4, pp. 592-602, 2015.

[20] S. H. Liang and S. Y. Shi, "Soliton solutions to Kirchhoff type problems involving the critical growth in $\mathbb{R}^{N}$," Nonlinear Analysis: Theory, Methods \& Applications, vol. 81, pp. 31-41, 2013.

[21] A. Canino, "Multiplicity of solutions for quasilinear elliptic equations," Topological Methods in Nonlinear Analysis, vol. 6, no. 2, pp. 357-370, 1995.

[22] A. Canino and M. Degiovanni, "Nonsmooth critical point theory and quasilinear elliptic equations," in Topological Methods in Differential Equations and Inclusions (Montreal, PQ, (1994)), vol. 472 of NATO Advanced Science Institutes Series C: Mathematical and Physical Sciences, pp. 1-50, Kluwer Academic Publishers, Dordrecht, The Netherlands, 1995. 


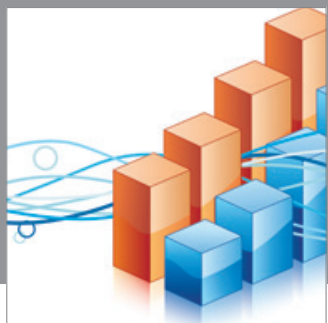

Advances in

Operations Research

mansans

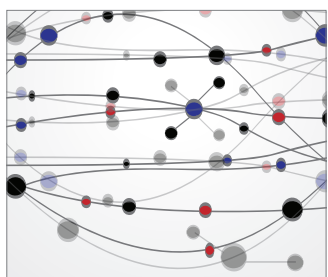

The Scientific World Journal
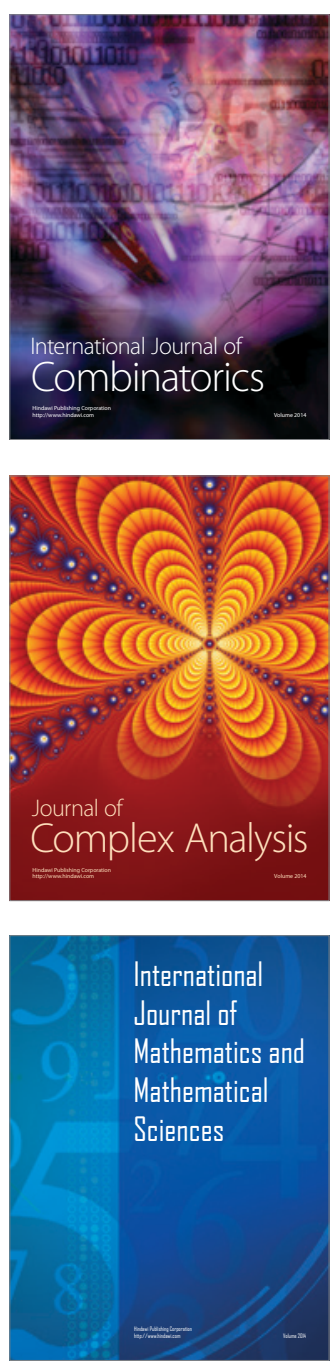
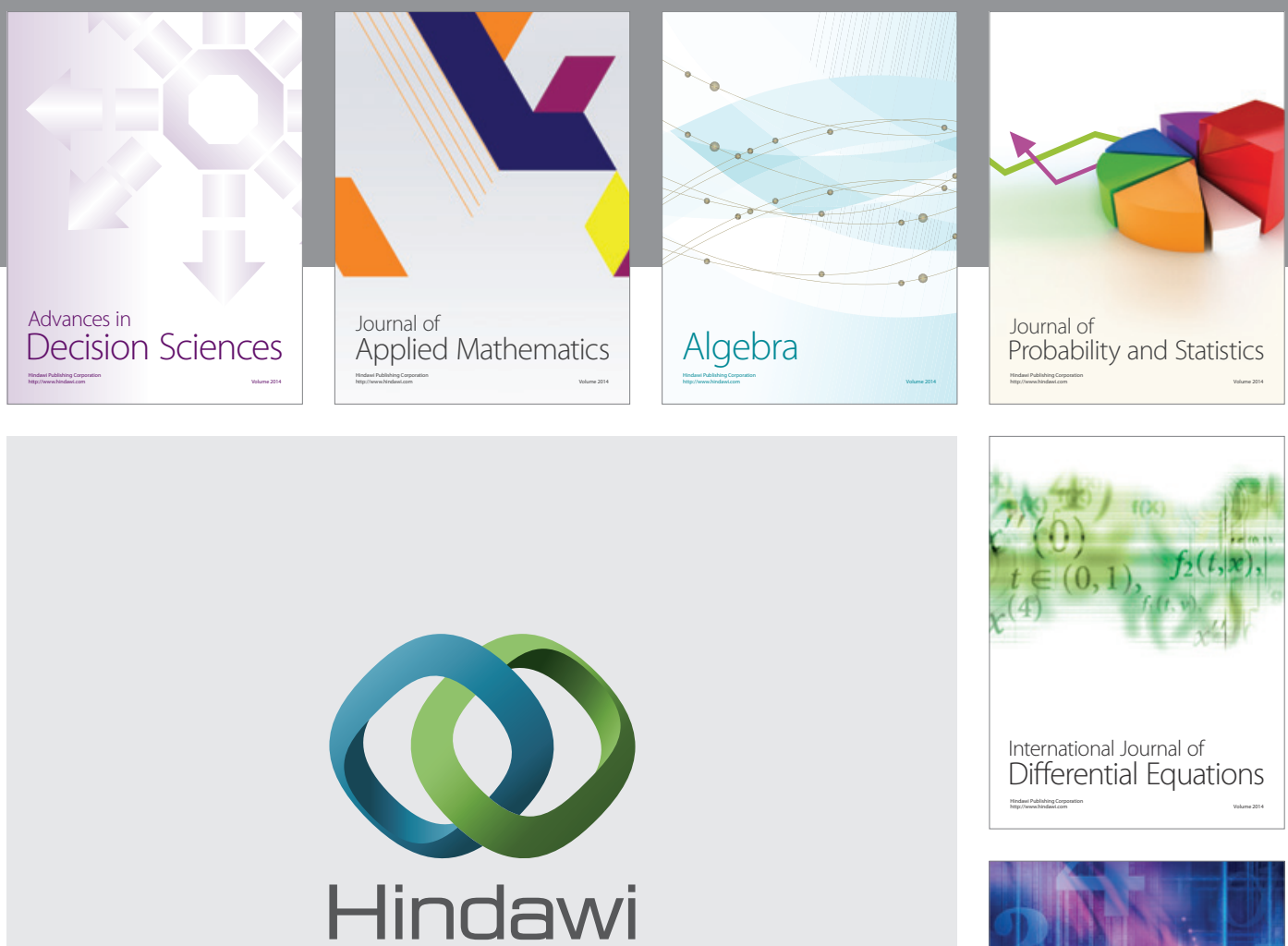

Submit your manuscripts at http://www.hindawi.com
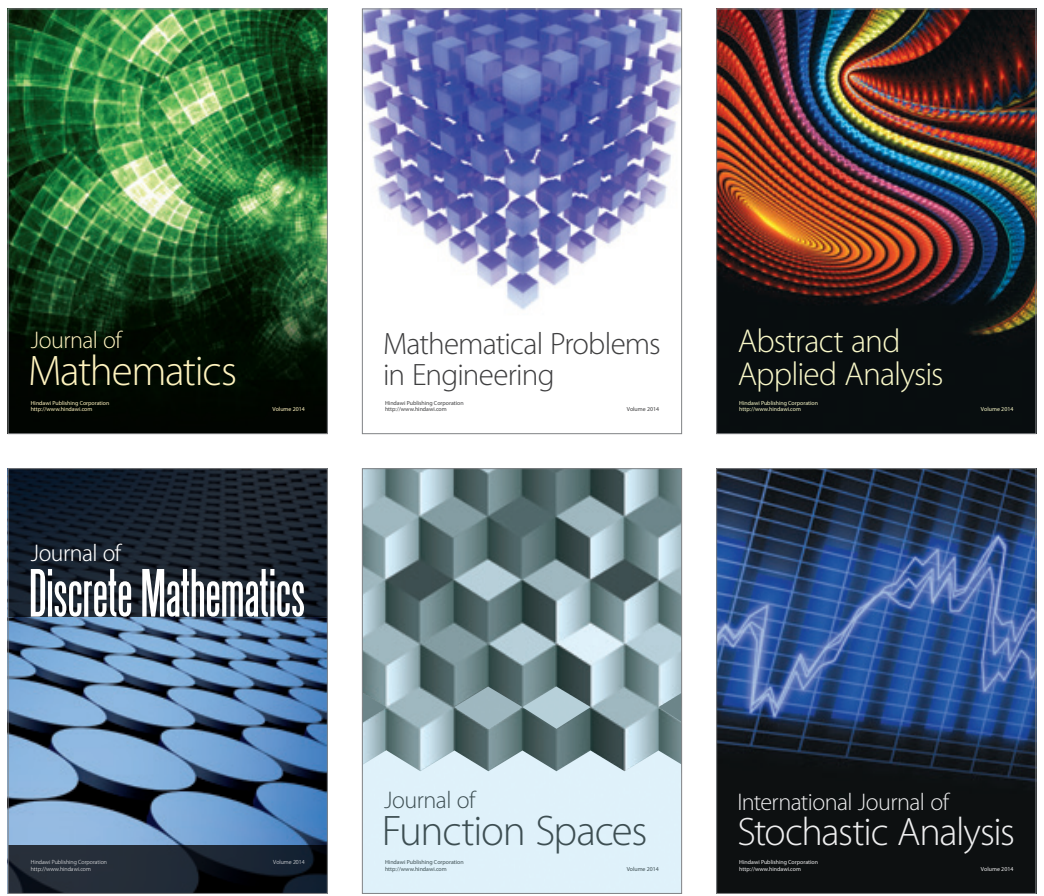

Journal of

Function Spaces

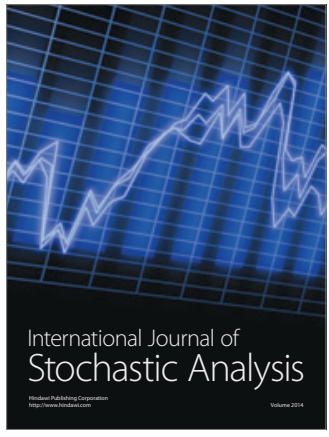

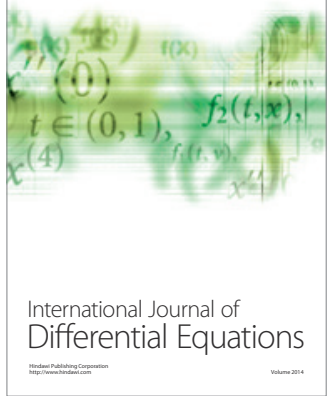
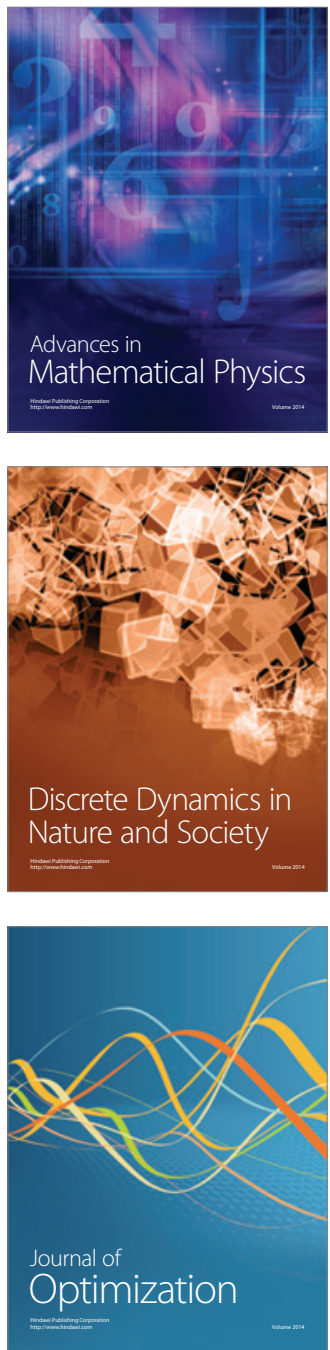